\title{
AUSTRALOMALOTYLUS RAGEAUI n. sp., ENCYRTIDAE PARASITE DE SARCOPHAGA sp. EN NOUVELLE-CALÉDONIE
}

\author{
Par Jean RISBEC
}

M. J. Rageau, entomologiste médical de l'Institut français d'Océanie, m'a adressé une série de Chalcidoïdes Encyrtidæ, qu'il a obtenus de pupes de Sarcophaga, et qui sont peut-être utilisables dans la lutte biologique contre certains Diptères nuisibles.

Il m'a été impossible de rapporter les exemplaires à une espèce, et même à un genre connu. Je suis donc amené à en publier la description.

\section{Description}

Genre : Australomalotylus n. g.

Diagnose. - Coloration non métallique, noire, et testacé plus ou moins rouge. Antennes de 11 articles, attachées près du clypeus. Scapes subcylindriques. 6 articles funiculaires. Massue de 3 articles, non tronquée. Ensemble du funicule et de la massue faiblement élargi de la base à l'apex. Pedicellus plus court que le premier funiculaire. Premiers articles funiculaires plus longs qu'épais, les derniers aussi longs qu'épais. Mandibules avec deux dents et un petit denticule supplémentaire. Palpes maxillaires longs et grêles, de 4 articles. Palpes labiaux de 3 articles. Pas de sillons parapsidaux. Réticulation très faible à la tête et au thorax, presque nulle au scutellum. Axillæ contigus sur l'axe. Une petite épine au milieu du bord antérieur du métathorax. Abdomen plus court que le thorax. Valves de la tarière courtes et larges. Ailes à franges courtes, à spéculum à peine indiqué par une rangée de soies spéciales, l'espace nu très réduit. Marginale égale à la postmarginale, plus longue que le radius. Pattes robustes.

Mâle comme la femelle, mais les antennes de 10 articles, plus longues, à articles densément pubescents et de longueur décroissant de la base à l'apex.

Ans. de Parasitologie, T. XXXI, N*1-2-1956. 


\section{Australomalotylus Rageaui $\mathrm{n}$. sp.}

Femelle. Coloration. - Tête et abdomen noirs. Thorax noir dorsalement, orangé latéralement et ventralement, avec des zones de passage allant des bords latéraux du pronotum aux métapleures. Prepectus brun roux. Metanotum et propodeum brun roux très foncé. Hanches et pattes entièrement rouge testacé. Antennes à radicule noir, scape rouge testacé, passant au noir à l'apex et brunissant à la base ; le reste de l'antenne noir. Tégulæ presque noirs. Les tarses sont plus ou moins brunis suivant les exemplaires.

Tête. - Longueur 0,25 (1), largeur 0,75 , hauteur 0,62 . Yeux : $0,37 \times 0,25$, distants de la bouche de 0,23 , luisants, à soies peu nombreuses, disséminées et relativement longues $(0,035)$. Les antennes sont à $0,06 \mathrm{du}$ clypeus, dont la limite est indiquée par un faible vallonnement. Ocelles situées à une distance des yeux à peu près égale à leur diamètre $(0,05)$, l'ocellum en triangle équilatéral.

Vertex assez large entre les yeux, 0,35. Fronto-vertex luisant, à très léger réseau fondamental et avec quelques points, peu profonds, disséminés. Face postérieure de la tête assez profondément concave, le bord net, mais non aigu. Soies noires, assez fortes, peu nombreuses, dressées. Les plus fortes sont aux tempes, le long du bord postérieur, et une rangée suivant les orbites. Sillons postantennaires formant une dépression en croissant demi-circulaire à bords largement arrondis. La sculpture est plus forte sur la partie du front inférieure aux antennes et sur les joues. Sillons génaux bien marqués. Cadre buccal très large; le bord du clypeus concave.

Antennes. - Radicule court. Scape : 0,28 , assez comprimé latéralement, mais peu élargi $(\max .0,06)$, un peu tordu vers l'apex, portant quelques soies à la face antérieure; trois plus fortes en particulier.

Pedicellus : $0,1 \times 0,05.1^{\text {er }}$ funiculaire : $0,125 \times 0,07$. A partir de là, l'épaisseur croît progressivement jusqu'au max. 0,08 , à la massue. La longueur des articles funiculaires décroît de 0,1 à 0,075 . Massue mesurant 0,175 , comprenant 3 articles de même longueur. Sur tous les articles, les soies noires, obliques, sont de l'ordre de 0,05 ; il y a quelques courts sensilli à la partie antérieure des articles funiculaires 2 à 7 . Articles bien séparès, en chapelet, à surface irrégulièrement mamelonnée.

Mandibules brun roux foncé, à surface rugueuse, relativement longues. Palpes rouge testacé, les labiaux beaucoup plus courts que les maxillaires.

(1) Toutes les mesures sont indiquées en millimètres. 


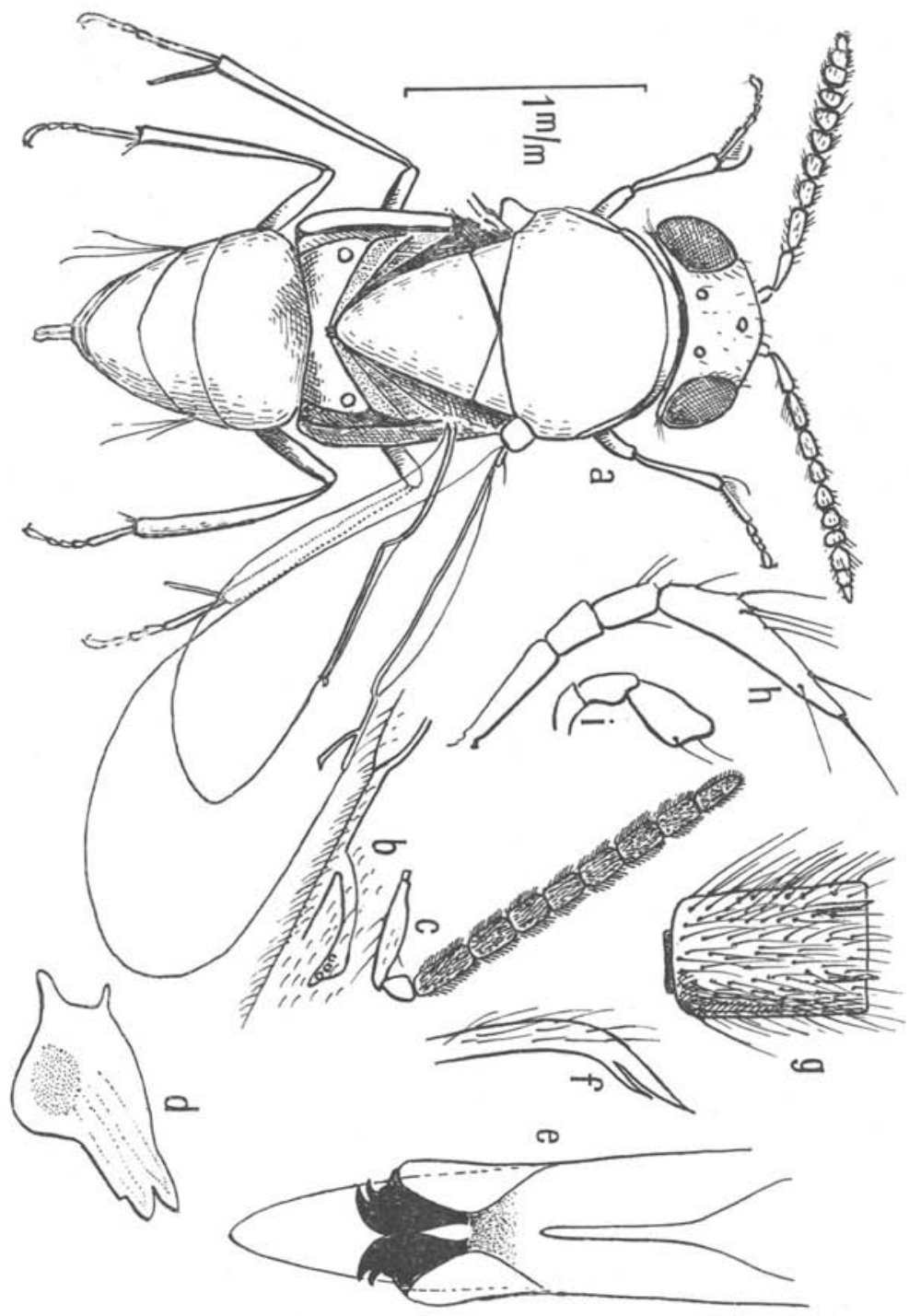

Australomalotylus Rageaui n. sp.

a) Femelle, dorsalement; $b$ ) nervures de la région marginale de l'aile antérieure ; $c$ ) antenne du mâle ; $d$ ) mandibule ; $e$ ) pénis ; $f$ ) extrémité de l'éperon tibial antérieur; $g$ ) $5^{e}$ article funiculaire de l'antenne màle $; h$ ) palpe maxillaire, gr. 250 ; i) palpe labial au même grossissement. 
Thorax. - Pronotum à peine visible dorsalement, couvert par la face postérieure de la tête. Mesonotum vaste : 0,55 , finement réticulé, rugueux, avec une prédominance transverse et des soies noires disséminées.

Scutellum : 0,55 , très luisant, surtout à la partie postérieure qui a un léger reflet cuivré. Le scutellum a des soies comme au mesonotum, moins nombreuses, mais plus fortes à la partie postérieure, les deux dernières surtout. Axillæ très faiblement réticulés.

Metanotum : 0,06, sur l'axe, dans un plan presque perpendiculaire à celui du scutellum. Sous l'apex du scutellum est la petite épine, bifide, du bord antérieur. Sur l'axe, en arrière de l'épine, est une bande lisse, surélevée, et qui s'élargit vers l'arrière. Le propodeum se développe latéralement, jusqu'à 0,2 de long, et se divise en deux parties égales, l'antérieure déprimée en gouttière à fond plat, la postérieure en bande plane surélevée. Les deux parties sont également réticulées, rugueuses.

Propodeum : 0,09 au milieu, étalé jusqu’à 0,25 latéralement, à surface presque lisse. Dans l'angle latéral antérieur est un vaste stigmate à bord circulaire.

Faces latérales du pronotum étroites, réticulées. Prepectus assez grand, à bord arrondi en avant des tegulæ, un peu déprimé sur sa moitié antérieure, faiblement réticuleux, rugueux. Mésopleures ovales, un peu tronqués près du prepectus, luisants, à peu près lisses, légèrement cannelés longitudinalement. Métapleures réduits, presque demi-circulaires, très légèrement réticulés.

Abdomen. - De longueur relative assez variable, mais toujours plus court que le thorax, subtriangulaire. Face dorsale à peu près plane, face ventrale très saillante en toit. Surface luisante, mais à très léger réseau, lequel disparaît à la partie antérieure des tergites. Valves de la tarière faisant saillie de 0,11 , larges et plates.

Ailes. - Légèrement enfumées. Ailes antérieures à franges de 0,03 . Soies discales nombreuses. Cellule costale : 0,82 , assez largement en segment de cercle ( $\max$. au milieu : 0,075 ), à soies nombreuses vers le bord libre. Marginale + postmarginale $=0,25$, subégales. Radius : 0,15 .

Ailes postérieures larges, à franges comme les antérieures. Nervures : $0,37,0,08$ (correspond à une courte courbure et à une épaisseur plus faible, avec base de la cellule qui s'effile jusque près des hamuli, 0,43.

Pattes. - Relativement robustes. Hanches antérieures : 0,32, assez épaisses. Trochanters : 0,1. Cuisses : 0,5 . Tibias : 0,38 , avec fort éperon de 0,125. Tarses : 0,37. 
Hanches moyennes : 0,37, présentant, près de l'apex, dorsalement, une épine qui s'applique sur l'articulation hanche-cuisse postérieure. Trochanters + cuisses : 0,8 . Tibias : 0,75 , à éperon de 0,25 . Tibias : 0,5 .

Hanches postérieures, presque aussi longues qu'épaisses à la base : 0,25 . Cuisses + trochanters : 0,57 ; les cuisses un peu élargies $(0,13)$, comprimées, courbées. Tibias : 0,6 , comprimés et courbés comme les cuisses, relativement courts : 0,5, grêles, avec un petit éperon de 0,1 et un second très réduit. Tarses : 0,4 .

Dimensions. - Taille : 2,47. Largeur du thorax : 0,82.

Abdomen. - Longueur : 0,95 ; largeur : 0,95 (valves de la tarière en plus).

Ailes antérieures : $2,12 \times 0,75$. Ailes postérieures : $1,5 \times 0,42$.

La taille des femelles varie de 2,25 à 2,47 .

MÂLE. Comme la femelle, mais la teinte générale un peu plus foncée. Les zones de passages sont rapprochées du ventre ; la base des mésopleures est brunie. Metanotum et propodeum complètement noirs. Abdomen un peu plus allongé. Antennes plus longues, avec 7 articles funiculaires séparés et une massue terminale indivise. Articles assez aplatis, à pubescence régulière, dense. La taille des articles décroît de 0,18 à 0,1, du $1^{\text {or }}$ au $7^{\circ}$ funiculaire. Massue terminale : 0,13 . L'épaisseur, à peu près constante, décroît assez irrégulièrement vers l'apex.

Taille : 2,4, dont 1,12 pour l'abdomen. Le plus petit exemplaire mesure 2,2 .

16 et 3 ơ, Nouméa, Institut français d’Océanie, J. Rageau.

\section{Discussion}

Qu'on utilise les tableaux de Girault, de Ferrière, d'Erdös et Novicky, on arrive toujours au genre Homalotylus. Le nouveau genre en diffère cependant parce que Homalotylus a des sillons postantennaires très faibles ou nuls, le pedicellus plus long que le premier funiculaire, la massue largement tronquée obliquement, la marginale très courte, la postmarginale presque nulle, la tarière cachée et des soies blanches abondantes au propodeum. Les colorations sont différentes.

Un genre voisin, Aztecencyrtus Timberlake, a les ocelles moins éloignés des yeux, les scrobes réduits, les antennes moins éloignées de la bouche, les premiers funiculaires réduits presque à des annelli, les mandibules terminés par trois fortes dents aiguës, les palpes labiaux de 3 articles, le thorax plan, le scutellum petit. 